\title{
Left Ventricular Systolic Dyssynchrony Index and Endothelial Dysfunction Parameters As Subclinical Predictors of Cardiovascular Involvement in Patients with Beta-Thalassaemia Major
}

\author{
Hatice Solmaz ${ }^{1}$, Ali Cabuk ${ }^{1}$, zeynep altın², esin özcan², and Oner Ozdogan 3 \\ ${ }^{1}$ Turkiye Cumhuriyeti Saglik Bakanligi Izmir Tepecik Egitim ve Arastirma Hastanesi \\ ${ }^{2}$ Affiliation not available \\ ${ }^{3}$ Tepecik Training and Research Hospital
}

January 21, 2021

\begin{abstract}
Objective: Cardiovascular involvement due to iron overload is the leading cause of morbidity and mortality in patients with betathalassaemia major ( $\beta-\mathrm{TM})$. However many patients remain asymptomatic until the late stage. In this study, we investigated the role of real-time three-dimensional echocardiography (RT3DE) findings and endothelial dysfunction parameters in asymptomatic $\beta$-TM patients, and the relationship between these parameters and cardiac magnetic resonance imaging (MRI) T2 * value. Methods: 51 asymptomatic $\beta$-TM patients who were receiving regular blood transfusions were evaluated by two-dimensional echocardiography (2DE) and RT3DE examinations including endothelial dysfunction parameters. The study population was divided into two subgroups based on their cardiac MRI T2* values (MRI T2* [?]20ms and $>20 \mathrm{~ms}$ ). The relationships between serum ferritin levels, 2DE and RT3DE measurements, endothelial dysfunction parameters, and cardiac MRI T2* values were investigated. Results: Although all left ventricle ejection fraction (LVEF) values obtained by 2DE were within normal limits ([?]50\%), they were not associated with MRI T2* values. LVEFRT3D $(53.25+2.33$ vs $58.81+1.02)$, SDI12 (6.53 + 0.56 vs 2.85 $+0.48)$, SDI16 $(7.65+0.75$ vs $3.26+0.49)$ were significantly different and negatively correlated between two groups respectively . Flow-mediated dilatation (FMD) $(6.08 \%+0.34$ vs $14.46 \%+1.12)$, aortic strain $(7.79 \%+2.19$ vs $12.76 \%+4.19)$, and serum ferritin values were significantly different and negatively correlated between two groups respectively. Conclusion: Decreased LVEF and increased SDI by RT3DE could be parameters of early cardiac deterioration. Decreased FMD and aortic strain may be good predictors of subclinical cardiovascular involvement in asymptomatic patients with $\beta$-TM.
\end{abstract}

Left Ventricular Systolic Dyssynchrony Index and Endothelial Dysfunction Parameters As Subclinical Predictors of Cardiovascular Involvement in Patients with Beta-Thalassaemia Major

\section{Abstract}

Background and Objective: Cardiovascular involvement due to iron overload is the leading cause of morbidity and mortality in patients diagnosed with beta-thalassaemia major $(\beta-T M)$. However many patients remain asymptomatic until the late stage. In this cross-sectional study, we investigated the role of real-time three-dimensional (RT3D) echocardiography findings and endothelial dysfunction parameters in asymptomatic $\beta$-TM patients, and the relationship between these parameters and cardiac magnetic resonanace imaging $(\mathrm{MRI}) \mathrm{T} 2 *$ value.

Methods: 51 asymptomatic $\beta$-TM patients who were receiving regular blood transfusions were evaluated by 
two-dimensional (2D) and RT3D echocardiography examinations including endothelial dysfunction parameters. The study population was divided into two subgroups based on their cardiac MRI T2* values (MRI T2* [?]20ms and $>20 \mathrm{~ms}$ ). The relationships between serum ferritin levels, 2D and RT3D echocardiography measurements, endothelial dysfunction parameters, and cardiac MRI T2* values were investigated.

Results: Among the echocardiographic findings, although all left ventricle ejection fraction (LVEF) values obtained by $2 \mathrm{D}$ echocardiography were within normal limits ([?]50\%), they were not associated with MRI T2* values. LVEFRT3D $(53.25+2.33$ vs $58.81+1.02)$, SDI12 $(6.53+0.56$ vs $2.85+0.48)$, and SDI16 $(7.65$ +0.75 vs $3.26+0.49)$ were significantly different and negatively corraleted between groups with MR1 T2* [?] $20 \mathrm{~ms}$ and $>20 \mathrm{~ms}$, respectively. Flow-mediated dilatation (FMD) $(6.08 \%+0.34$ vs $14.46 \%+1.12)$, aortic strain $(7.79 \%+2.19$ vs $12.76 \%+4.19)$, and serum ferritin values were significantly different and negatively corraleted between groups with $\mathrm{T} 2 *[?] 20 \mathrm{~ms}$ and $>20 \mathrm{~ms}$, respectively.

Conclusion: Decreased LVEF and increased SDI by RT3D echocardiography could be earlier parameters of early cardiac deterioration. Decreased FMD and aortic strain may be good predictors of sublinical cardiovascular involvement in asymptomatic patients with $\beta$-TM.

Key words: Beta-thalassaemia major, Cardiovascular involvement, Endothelial dysfunction parameters, Left ventricular systolic dyssynchrony index, Real-time 3D echocardiography

\section{1.İntroduction}

Thalassemia is one of the most common genetic disorders worldwide (1). According to the severity, beta thalassemia major $(\beta-T M)$ is the most severe form; and affected patients exhibit symptoms within the first year of life and requires lifelong regular blood transfusion therapy for survival (2). Because standard transfusion therapy adds $0.32-0.64 \mathrm{mg} / \mathrm{kg} /$ day of iron to the body and causes iron overload, patients need adequate iron-chelating treatment to prevent its progressive accumulation in different organs, including the cardiovascular system (3). However, cardiovascular involvement and deterioration in endothelial function cannot be completely overcome even with iron chelation therapy guided by accurate and strict monitoring. Ultimately, the cardiovascular condition caused by iron overload remains the leading cause of death in $\beta$-TM patients $(4,5)$. Therefore, the early detection of conditions caused by cardiovascular iron overload is an important clinical need to prevent or diagnose and treat complications, improve prognosis (6).

In this study, we aimed to identify subclinical predictors of cardiovascular involvement like left ventricular systolic dyssynchrony index (SDI), flow mediated dilatation (FMD) and aortic strain in patients with $\beta$-TM.

\section{Materials and Methods}

\subsection{Study population}

In this cross-sectional study, a total of 51 asymptomatic participants aged between 18 and 45 with $\beta$-TM on regular follow-up were recruited from the haematology clinic. We did not enroll a healty control group. Instead of this, we defined two groups based on their magnetic resonance (MR) T2* values (as [?]20 msec and $>20 \mathrm{msec}$ ) which is an accepted indicator of cardiovascular involvement. Patients with coronary heart disease, moderate to severe valvular heart disease, congenital heart disease, atrial fibrillation, risk factors for atherosclerosis (hypertension, diabetes, hyperlipidemia, family history and smoking), left ventricular (LV) systolic or diastolic dysfunction, and patients who were using drugs which effect cardiovascular function were excluded from the study. Haematological and biochemical parameters were measured. As anemia can affect cardiac hemodynamic parameters, cardiovascular assessment was performed after transfusion to minimize potential confounding influence of anaemia.

We measured serum ferritin levels, and performed two- dimensional echocardiography (2DE) and real-time three-dimensional echocardiography (RT3DE) at the same session during rest condition. We analyzed T2* cardiac magnetic resonance imaging for determination of myocardial iron load, and assessed endothelial functions by brachial artery flow-mediated dilatation (FMD) and aortic strain. 
The Local Ethics Committee approved this study and the informed consents were obtained from all participants.

\subsection{Echocardiographic studies and image acquisition}

The device used for conventional 2DE and RT3DE was the Philips IE33 system with a matrix array ultrasonographic transducer (X4.1 transducer; Philips Medical Systems, Bothell, WA, USA). We used Simpson's biplane method for measurement of ejection fraction with in an apical 4 and 2 chamber views according to recent guidelines (7). After endocardial tracing of borders at end systole and end diastole was performed, we calculated LVEF with the formula by using LV end-diastolic (EDV) and LV end-systolic volumes (ESV) as follows: $\mathrm{EF}=(\mathrm{EDV}-\mathrm{ESV}) / \mathrm{EDV}$.

To achieve optimal Real-time 3D echocardiographic acquisition of the left ventricle, images were optimized by managing the gain, compress, and time-gain compensation controls. Sector width and depth at two dimensional setting and we switched to xPlane imaging to detect the quality of endocardial borders at orthogonal view. After obtaining a satisfactory image which included all segments of myocardium clearly, full-volume acquisition with capturing of 4 adjacent ECG gated sub-volumes over 4 consecutive cardiac cycles in a pyramidal scan was performed during patients end-expiratory apnea within single breathhold to prevent stitching artifacts. Acquisition of each sub-volume was triggered to the ECG R-wave of every other heartbeat to allow sufficient time for the probe to be recalibrated and each subvolume stored.

Post processing analysis of volumes and EF were performed using Qlab software (Version 9.0; Philips Medical Systems) by two expert echocardiographer with extensive training in 3D echocardiography. The multiplanar view was aligned to maximize the LV cavity long- and short-axes in the 2- and 4-chamber views. End- diastole was defined as the first frame after mitral valve closure and end- systole was defined as the first frame after aortic valve closure. After first identifying the apex and mitral annulus on the end-diastolic and end-systolic frames, transverse plane was adjusted at the level of papillary muscles and saggital plane was adjusted from the midline of mitral annulus to apex. Then automatic endocardial border definition was performed by applying 4 mitral annular and 1 apical points: septal, lateral, anterior, inferior annular, and apex (Figure 1). Manual corrections of the LV endocardial borders including papillary muscles and trabeculations were performed if the operator judges the automatically detected surface as suboptimal. Sequence analysis were performed and checked for correct border detection frame- by- frame. At the end of analysis LVEDV, LVESV, LVEF, stroke volume, cardiac output, 16 segments and 12 segments SDI were obtained (Figure 2) (8).

\subsection{Assessment of aortic strain}

Aortic root, AoS and AoD was measured by 2D motion mode (M-mode) transthoracic echocardiography at a level of 3-4 cm above the aortic valve from a transthoracic parasternal long-axis view, at the time of maximum aortic anterior motion, and at the peak of the QRS complex at the simultaneous electrocardiogram recording, respectively. The percentage change in the aortic root was calculated as DAo\% $=100 \times($ AoS AoD) / AoD to obtain aortic strain (9).

\subsection{Assessment of brachial artery endothelial function}

To evaluate the endothelial function of the brachial artery noninvasively, flow-mediated endotheliumdependent vasodilation was assessed by measuring the brachial artery diameter at baseline and during reactive hyperemia. All patient fasted for at least $8 \mathrm{~h}$ and avoided consuming caffeine or smoking cigarettes for $12 \mathrm{~h}$ prior to their vascular examination. All vasoactive medications were withheld 8 hours before the scan. Each patient were rested in a supine position for $30 \mathrm{~min}$ and remained in a quiet, air-conditioned room, with its temperature kept between 20 and $22 *$ C. All studies were performed using an ultrasound system (Philips EPIQ 7C, Philips Healthcare, Andover, MA, USA) with a broadband linear array transducer with a 3-12 MHz range (Philips L12-3). The brachial artery was viewed longitudinally $5 \mathrm{~cm}$ above antecubital fossa and FMD was assessed according to the existing guidelines(10).

When the clearest image of the anterior and posterior intimal interfaces between the lumen and vessel wall was obtained, baseline diameter measurements were taken at the end of the diastole (timed by the peak 
of $\mathrm{R}$ wave on electrocardiogram) for [?] 3 times. Before starting measurement, cuff was fitted distally to the brachial artery. After baseline measurement, the cuff was inflated to at least $50 \mathrm{~mm} \mathrm{Hg}$ above systolic pressure to occlude arterial inflow for 5 minutes. The longitudinal image or artery was scanned continuously from $30 \mathrm{~s}$ before to 90 seconds after cuff deflation and the maximal diameter of the artery was defined during reactive hyperemia. The basal diameter was defined as the average of all measures collected before inflation, and FMD was calculated as the percentage change in peak vessel diameter from the baseline value following cuff deflation: peak diameter-baseline diameter) / baseline diameter. In present study, according to the results of previous studies, FMD value under $10.0 \%$ was accepted as endothelial dysfunction $(11,12)$.

\subsection{Magnetic Resonance Imaging}

MRI examinations were performed on a GE 1.5 tesla MR device. MRI scans were used to measure cardiac T2* which is an MRI parameter representing relaxation, as well as myocardial iron load. Imaging was acquired in a mid papillary shot axis during the late cardiac cycle diastole phase in a single breath-hold with ECG gating and cardiac T2* time was measured at mid-septum; [?]20 msec was accepted abnormal myocardial iron load (13).

\section{Statistics}

Statistical analyses were performed using the SPSS software version 24. The variables were investigated using visual (histograms, probability plots) and analytical methods (Kolmogorov-Smirnor/Shapiro-Wilk's test) to determine whether or not they are normally distributed. Descriptive analyses were presented using means and standart deviations for normally distributed variables, and using medians and interquartile range for the non-normally distributed variables. Student's t-test was used to compare parameters which were normally distributed, and the correlation coefficients and their significance were calculated using the Pearson test. Mann-Whitney U test was used to compare paramaters which were non-normally distributed, and the correlation coefficients and their significance were calculated using the Spearman test. A $5 \%$ type-I error level was used to infer statistical significance.

\section{Results}

27 of 51 participants were female $(52.9 \%)$ and mean ages of two study population were similar $(27.66+$ 1.62 vs $27.13+1.01$ for patients with $\mathrm{T}^{*}$ [?] $20 \mathrm{~ms}$ and $>20 \mathrm{~ms}$, respectively). Baseline characterisitcs of study population shown in Table 1 . We found that LVEF3D $(53.25+2.33$ vs $58.81+1.02)$, SDI12 $(6.53$ +0.56 vs $2.85+0.48)$, SDI16 $(7.65+0.75$ vs $3.26+0.49)$, FMD $(6.08 \%+0.34$ vs $14.46 \%+1.12)$, aortic strain $(7.79 \%+2.19$ vs $12.76 \%+4.19)$; and also serum ferritin values were significantly different between groups with $\mathrm{T} 2 *[?] 20 \mathrm{~ms}$ and $>20 \mathrm{~ms}$, respectively (Table 2 ). For all parameters found significantly different between two study groups, there were also significant positive or negative correlations with $\mathrm{T}^{*}$ value (Figure 3). Echocardiographic measurements were performed by two experienced operators and repeated by same operator at another time; and perfect inter-observer $(\mathrm{k}=0.89)$ and intra-observer $(\mathrm{k}=0.92)$ agreements were achieved.

\section{Discussion}

In this study, we evaluated the relationship between echo parameters of subclinical myocardial dysfunction, endothelial dysfunction parameters, and MRI T2 ${ }^{*}$ values which is the gold standard for cardiac iron deposition.

Among the measured variables, all LVEF values obtained with 2DE were normal ([?]50\%) and not significantly associated with MRI T2* values. This finding is in agreement with previous studies which indicates its inefficiency in predicting abnormal cardiac iron deposition (14). This is likely due to limitations of 2DE compared to 3DE for measuring LVEF which lead to systematic overestimation of volumes and LVEF2D (15). On the other hand, since echocardiographic measurement of LVEF with 2DE may be inaccurate in 
patients with regional wall motion abnormalities or mechanical dyssynchrony, precence of heart failure can not be ruled out even with normal LVEF2D (15).

In our study, in contrast to other published studies $(16,17)$, levels of serum ferritin were significantly different between two groups and negatively correlated with MRI T2*. Our finding is similar to Wahidiyat et al. large-scale population-based- study which found statistically significant correlation between serum ferritin level and liver and cardiac T2* (18). This may indicate an ongoing remodeling process even in patients with preserved LVEF2D and it is important to identify patients while their cardiomyopathy is still reversible.

In the present study, while LVEF2D was preserved and not significantly associated with MRI T2*, LVEF3D, SDI12 and SDI16 was significantly different between two groups and negatively correlated with MRI T2* findings. These relationships suggest that iron overload may play an important role in the pathogenesis of ventricular dyssynchrony $(19,20)$. In light of evidence, we know that ventricular dysynchrony has an independent mechanism of decrease in left ventricular systolic function and is an important parameter in determining patients with severely decreased left ventricular systolic function (21-23). Furthermore Bae et al. demonstrated a significant improvement of ventricular dysynchrony after the antihypertensive treatment when compared to the baseline ventricular dysynchrony in hypertensive patients with preserved LV systolic function (24). These findings show importance of properly assessment of cardiac function particularly in this population.

While heart disease is the main cause of death in $\beta$-TM, vascular endothelial pathologies as well as myocardial parenchymal injury has been reported as the one of the main cause of cardiovascular complications $(25,26)$. Its known that, vascular bed function and integrity were affected by chronic hemolysis because both hemolysis and iron overload are the main causes of oxidative damage in $\beta$-TM patients. Plasma-free hemoglobin, released as a result of chronic intravascular hemolysis, directly causes the formation of reactive oxygen species that catalyze oxidative damage in vascular cellular structures. In addition, it has been observed that free hemoglobin reacts with NO, causes NO deactivation and limits NO diffusion from the endothelium to smooth muscle cells (27). Similarly, the release of erythrocyte arginase during intravascular hemolysis may limit the cellular availability of L-arginine, a substrate for NO synthesis, and cause insufficient NO production (28). This imbalance between NO production and consumption causes a decrease in NO bioavailability and ultimately leads to the development of endothelial dysfunction by inhibiting vasodilation (29). Endothelial dysfunction probably contributes in part to the increase in arterial stiffness. Not surprisingly, flow-mediated dilatation of the brachial artery and aortic strain are important research tools for assessing vascular biology and endothelial function and detects early stages of atherosclerosis. $(30,31)$. In the present study we found that FMD and aortic strain values were significantly different between two groups and negatively colerated with T2* values. Stakos et al. (32) showed in their study that, patients with $\beta$-TM and normal cardiac iron levels documented by $\mathrm{T} 2 *$ and no clinical signs of cardiac dysfunction, have also increased aortic stiffness compared with normal control subjects. FMD was found significantly impaired in patients with $\beta$-TM compared with healty control group in another study (33). While our findings may lead to the hypothesis that the aorta and peripheral arteries exhibit an accelerated aging rate in negative correlation with cardiac $\mathrm{T} 2$ values, this group of patients, including patients with normal T2 findings, may undergo a lifelong process of vascular remodeling. Knowledge about the complexity of the underlying mechanisms can help prevent or treat potential complications.

\section{Conclusion}

We found that decreased LVEF3D and increased SDI, and also endothelial dysfunction shown by decresed FMD of brachial artery and aortic strain, when used together with MR T2* values, may be good predictors of sublinical cardiovascular involvement in patients with $\beta$-TM. Prospective large-scale studies are needed to determine whether these parameters can be used as subclinical determinants of cardiovascular iron overload.

Conflict of Interest: None declared

Tables: 
Table 1 . Baseline characteristics of study population.

Table 2. Three dimensional echocardiography measurements, endothelial dysfunction parameters and ferritin levels of participants.

Figure Legends:

Figure 1. LV end-diastolic (A) and end-systolic (B) endocardial border tracings in full volume analysis for LVEF and LV volumes measurement

Figure 2. Report page sample shows $16 / 12$ segments SDI results after sequential analysis.

Figure 3. Correlation graphics between T2* level and LVEF3D, aortic strain, FMD, serum ferritin levels, SDI16, SDI12. Left sided three graphics show positive correlations; and right sided three graphics shows negative correlations as expected.

\section{Autor Contrubitions:}

Design: Hatice Solmaz, Oner Ozdogan

Data collection: Hatice Solmaz, Zeynep Altin, Esin Albudak Ozcan

Data Analysis: Hatice Solmaz, Ali Kemal Cabuk

Drafting Article: Hatice Solmaz

Statistics: Zeynep Altin, Ali Kemal Cabuk

Critical revision of article: Oner Ozdogan

\section{References:}

1. Weatherall DJ: Thalassemia in the Next Millennium: Keynote Address a. Annals of the New York Academy of Sciences 1998:850:1-9.

2. SL Thein: Genetic modifiers of beta-thalassemia. Haematologica 2005:90:649-660.

3. Dubey AP, Parakh A, Dublish S: Current trends in the management of beta thalassemia. The Indian Journal of Pediatrics 2008:75:739.

4. Modell B, Khan M, Darlison M, et al: Improved survival of thalassaemia major in the UK and relation to T2* cardiovascular magnetic resonance. Journal of Cardiovascular Magnetic Resonance 2008:10:42.

5. Rooyakkers TM, Stroes ESG, Kooistra MP, et al: Ferric saccharate induces oxygen radical stress and endothelial dysfunction in vivo. European Journal of Clinical Investigation 2002:32:9-16.

6. Murphy CJ, Oudit GY: Iron-overload cardiomyopathy: pathophysiology, diagnosis, and treatment. Journal of Cardiac Failure 2010:16:888-900.

7. Lang RM, Badano LP, Mor-Avi V, et al: Recommendations for cardiac chamber quantification by echocardiography in adults: an update from the American Society of Echocardiography and the European Association of Cardiovascular Imaging. European Heart Journal-Cardiovascular Imaging 2015:16:233-271.

8. Kapetanakis S, Kearney MT, Siva A, et al: Real-time three- dimensional echocardiography: a novel technique to quantify global left ventricular mechanical dyssynchrony. Circulation 2005:112:992-1000.

9. Kasikcioglu HA, Karasulu L, Durgun E, et al: Aortic elastic properties and left ventricular diastolic dysfunction in patients with obstructive sleep apnea. Heart and Vessels 2005:20:239-244.

10. Corretti MC, Anderson TJ, Benjamin EJ, et al: Guidelines for the ultrasound assessment of endothelialdependent flow-mediated vasodilation of the brachial artery: a report of the International Brachial Artery Reactivity Task Force. Journal of the American College of Cardiology 2002:39:257-265. 
11. Bots ML, Westerink J, Rabelink TJ, et al: Assessment of flow-mediated vasodilatation (FMD) of the brachial artery: effects of technical aspects of the FMD measurement on the FMD response. European Heart Journal 2005:26:363-368.

12. Vogel RA: Measurement of endothelial function by brachial artery flow-mediated

vasodilation. The American Journal of Cardiology 2001:88:31-34.

13. Pennell DJ: T2* magnetic resonance and myocardial iron in thalassemia. Annals of the New York Academy of Sciences 2005:1054:373-378.

14. Moussavi F, Ghasabeh MA, Roodpeyma S, et al: Optimal method for early detection of cardiac disorders in thalassemia major patients: magnetic resonance imaging or echocardiography? Blood Research 2014:49:182-186.

15. Pennell DJ, Udelson JE, Arai AE, et al: American heart association committee on heart failure and transplantation of the council on clinical cardiology and council on cardiovascular radiology and imaging. Cardiovascular function and treatment in $\beta$-thalassemia major: a consensus statement from the american heart association. Circulation 2013:128:281-308.

16. Abtahi F, Abdi A, Jamshidi S, et al: Global longitudinal strain as an Indicator of cardiac Iron overload in thalassemia patients. Cardiovascular Ultrasound 2019:17:24.

17. Eghbali A, Taherahmadi H, Shahbazi M, et al: Association between serum ferritin level, cardiac and hepatic T2-star MRI in patients with major $\beta$-thalassemia. Iranian journal of Pediatric Hematology and Oncology 2014:4:17.

18. Wahidiyat PA, Liauw F, Sekarsari D, et al: Evaluation of cardiac and hepatic iron overload in thalassemia major patients with $\mathrm{T}^{*}$ magnetic resonance imaging. Hematology 2017:22:501-507.

19. Kim E, Giri SN, Pessah IN: Iron (II) is a modulator of ryanodine-sensitive calcium channels of cardiac muscle sarcoplasmic reticulum. Toxicology and Applied Pharmacology 1995:130:57-66.

20. Mavrogeni SI, Markussis V, Kaklamanis L, et al: A comparison of magnetic resonance imaging and cardiac biopsy in the evaluation of heart iron overload in patients with $\beta$-thalassemia major. European Journal of Haematology 2005:75:241-247.

21. Penicka M, Bartunek J, De Bruyne B, et al: Improvement of left ventricular function after cardiac resynchronization therapy is predicted by tissue Doppler imaging echocardiography. Circulation 2004:109:978-983.

22. Leclercq C, Faris O, Tunin R, et al: Systolic improvement and mechanical resynchronization does not require electrical synchrony in the dilated failing heart with left bundle-branch block. Circulation 2002:106:1760-1763.

23. Liodakis E, Al Sharef O, Dawson D, et al: The use of real-time three- dimensional echocardiography for assessing mechanical synchronicity. Heart 2009:95:1865-1871.

24. Bae BS, Kim KJ, Park JG, et al. Improvement in left ventricular systolic dyssynchrony in hypertensive patients after treatment of hypertension. Korean Circulation Journal 2011:41:16-22.

25. Borgna-Pignatti C, Cappellini MD, De Stefano P, et al: Survival and complications in thalassemia. Annals of the New York Academy of Sciences 2005:1054:40-47.

26. Cappellini MD: Prevalence of thromboembolic events among 8,860 patients with thalassaemia major and intermedia in the Mediterranean area and Iran. Thromb Haemost 2006:96:488-91.

27. Minneci PC, Deans KJ, Zhi H, et al: Hemolysis-associated endothelial dysfunction mediated by accelerated NO inactivation by decompartmentalized oxyhemoglobin. The Journal of Clinical Investigation 2005:115:3409-3417. 
28. Morris CR, Kato GJ, Poljakovic M, et al: Dysregulated arginine metabolism, hemolysis-associated pulmonary hypertension, and mortality in sickle cell disease. JAMA: Journal of the American Medical Association 2005:294:81-90.

29. Hahalis G, Kremastinos DT, Terzis G, et al: Global vasomotor dysfunction and accelerated vascular aging in $\beta$-thalassemia major. Atherosclerosis 2008:198:448-457.

30. Heitzer T, Schlinzig T, Krohn K, et al: Endothelial dysfunction, oxidative stress, and risk of cardiovascular events in patients with coronary artery disease. Circulation 2001:104:2673-2678.

31. Mancini GJ, Dahlof B, Diez J: Surrogate markers for cardiovascular disease: structural markers. Circulation 2004:109:IV-22-IV-30

32. Stakos DA, Margaritis D, Tziakas DN, et al: Cardiovascular involvement in patients with $\beta$-thalassemia major without cardiac iron overload. International Journal of Cardiology 2009:134:207-211.

33. Gupta A, Kapoor A, Phadke S, et al: Use of strain, strain rate, tissue velocity imaging, and endothelial function for early detection of cardiovascular involvement in patients with beta-thalassemia. Annals of Pediatric Cardiology 2017:10:158. 